\title{
Scintillation crystal growth at the CUP
}

\author{
SeJin $\mathrm{Ra}^{1}$, Cheol Ho Lee, Ju Kyung Son, Keon Ah Shin, Jun Seok Choe, Dae Yeon \\ Kim, M. H. Lee, W. G. Kang, D. Leonard, J. H. So. H. S. Lee, Y. D. Kim \\ Center for Underground Physics, Institute for Basic Science (IBS), \\ Daejeon 34126, Korea \\ E-mail: nsjneweibs.re.kr

\section{H. K. Park} \\ Department of Accelerator Science, Korea University, \\ Sejong 30019, Korea

\section{H. J. Kim} \\ Department of Physics, Kyungpook National University, \\ Daegu 41566, Korea
}

\begin{abstract}
The Center for Underground Physics (CUP) at the Institute for Basic Science (IBS) has been conducting two major experiments, the COSINE experiment for dark matter search and the AMoRE experiment for neutrinoless double beta decay search. The COSINE experiment is using NaI:Tl scintillation crystals and the AMoRE is studying the ${ }^{100} \mathrm{Mo}$ based scintillation crystals such as $\mathrm{CaMoO}_{4}(\mathrm{CMO}), \mathrm{Li}_{2} \mathrm{MoO}_{4}(\mathrm{LMO})$, etc. In order to develop ultra-pure scintillation crystals for the two experiments, we have built a clean-environment facility to minimize external contamination during the crystal growth. Here, we report the crystal growth facility and the internal background levels of the materials and grown crystals.
\end{abstract}

The 39th International Conference on High Energy Physics (ICHEP2018)

4-11 July, 2018

Seoul, Korea

\section{${ }^{1}$ Speaker}




\section{Introduction}

The CUP is currently conducting two major experiments: the COSINE is using NaI:Tl scintillating crystal for a Weakly Interacting Massive Particle (WIMP, a strong candidate of dark matter) search and the Advanced Mo-based Rare process Experiment (AMoRE) is using Mo based scintillating crystals for neutrinoless double beta decay search (a characterization of the neutrino) of ${ }^{100} \mathrm{Mo}$. In order to use scintillation crystals for the two experiments, we have to meet requirements such as scintillation light yield and ultra-low internal-background of the crystals. We, therefore, have been performing tests to optimize the growth condition for each type of crystals and planning crystal growths using highly purified powders.

We report on crystal growing facilities in the CUP and impurities in materials and grown crystals.

\section{Crystal growing facilities}
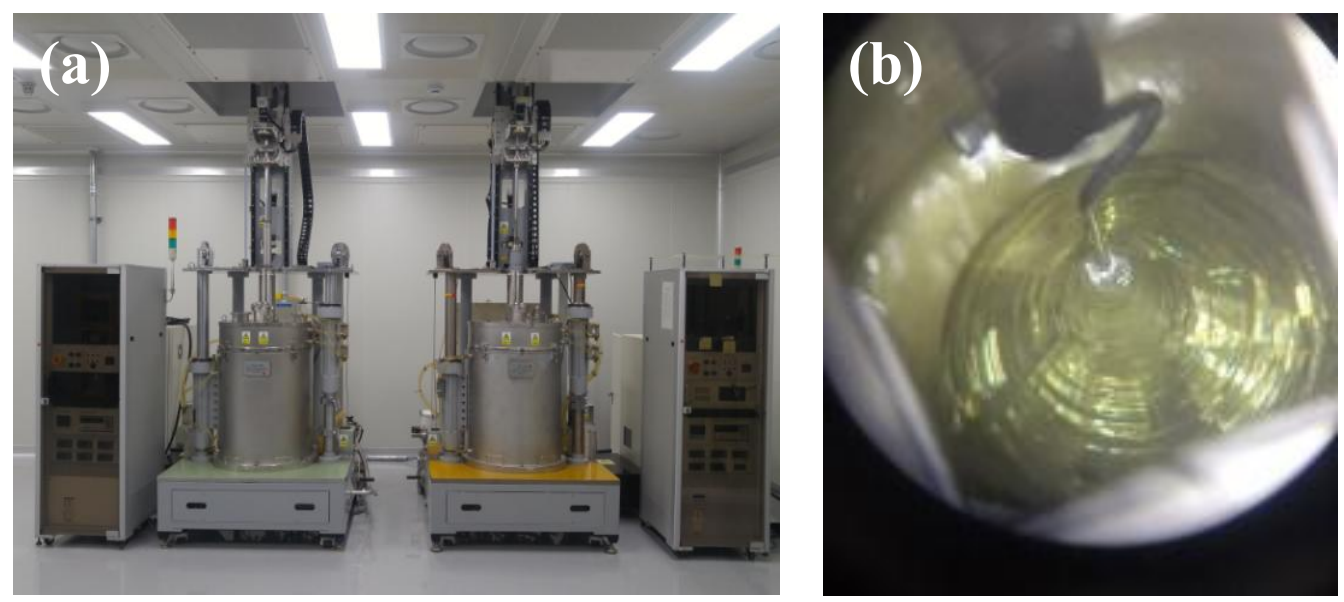

Figure 1: (a) Two full-size Czochralski growers for Mo based crystals, (b) an LMO crystal being grown.

\subsection{Growers for growing crystals}

We have two independant crystal growing facilities for NaI:Tl and $\mathrm{MoO}_{3}$ based crystals.

The facility for growing Mo based crystals is controlled by an air circulation system with HEPA filters to keep a class 10,000 environment for contamination-free (radioactivity and impurity) crystal growth. Two full-size Czochralski growers were installed to grow crystals for the AMoRE. A 'high-resolution load cell' is used to measure crystal weight for an automatic growing process. Due to this device, the diameter of the crystal can be maintained by controlling the temperature during the growth. Fig. 1 (a) and (b) show full-size Czochralski growers and a $\mathrm{Li}_{2} \mathrm{MoO}_{4}$ crystal being grown in a grower.

For NaI:Tl crystal growth, a dust collector and a ventillation system are installed in the facility to remove $\mathrm{NaI}$ (corrosive to metal) and $\mathrm{Tl}$ (toxic to human) vapors generated during the growth. More severe safety and cleanliness are considered for the NaI:Tl growing facility because of the personal safety. Fig. 2 (a) and (b) show two Kyropoulos growers to grow NaI:Tl crystals. Fig. 2 (a) is an R\&D grower to optimize the growth condition such as rotation speed and pulling 
rate. Fig. 2 (b) is a grower for full-size NaI:Tl crystals of $150 \mathrm{~kg}$. Both growers have resistance heaters and covered by refractories to prevent corrosion. Both of two different-size growers have the same internal structure to minimize the growth condition difference.

\subsection{Raw materials in the growing}

A CaCO $3\left(99.95 \%\right.$, Alfa Aesar) or $\mathrm{Li}_{2} \mathrm{CO}_{3}\left(99.998 \%\right.$, Alfa Aesar) and $\mathrm{MoO}_{3}(99.95 \%$, Alfa Aesar) as raw powders were mixed evenly with molar ratio of 1:1. Mixed powders were charged into a crucible. CMO crystal was grown in an iridium crucible and LMO in a platium one with a dimension of $\varnothing 100 \mathrm{~mm} \times \mathrm{H} 100 \mathrm{~mm}$. Alumina refractories were stacked and covered the crucible.

In order to grow NaI:Tl crystal, commercial-grade NaI (99.99 \%, Merck) and Tl (99.999 \%, Sigma Aldrich) were used for the test growing. Only $0.1 \mathrm{~mol} \%$ of $\mathrm{Tl}$ was mixed as a dopant and the raw powders were charged into a quartz crucible $(\varnothing 120 \mathrm{~mm} \times \mathrm{H} 150 \mathrm{~mm})$ to grow a crystal of $1.7 \mathrm{~kg}$ weight.
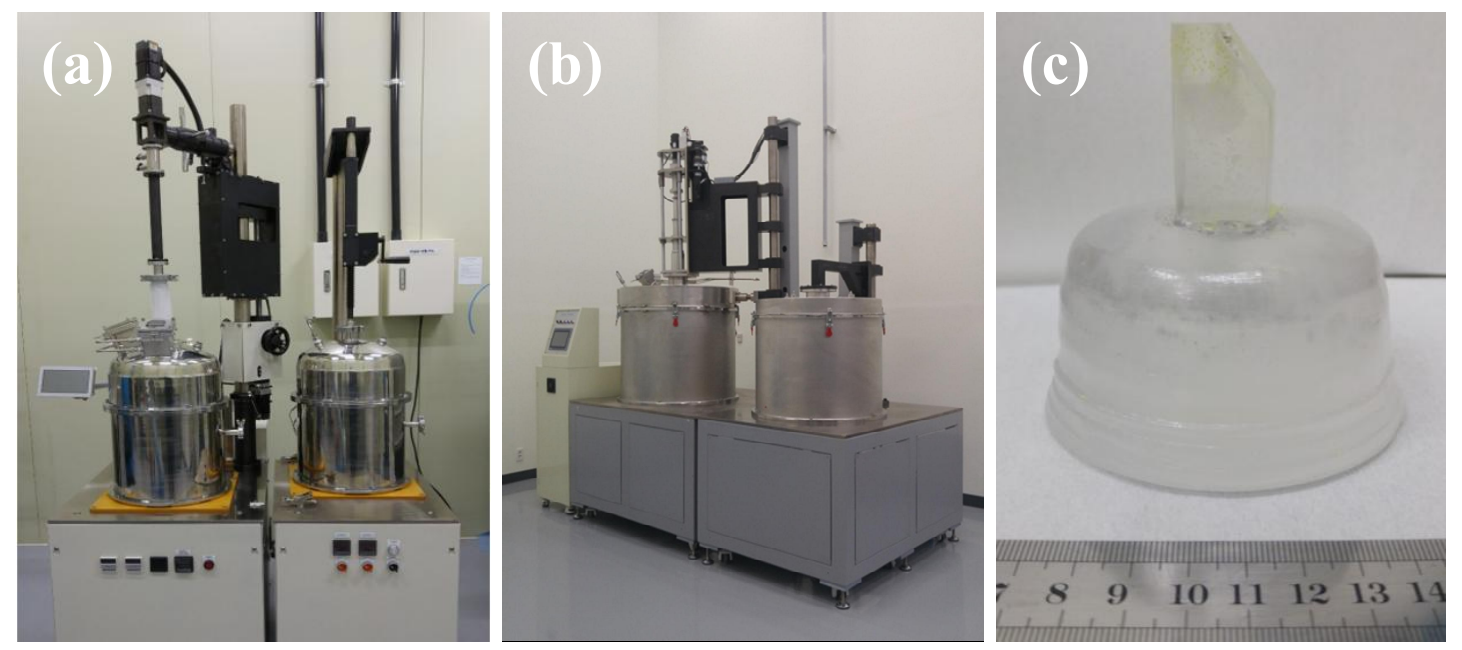

Figure 2: Two Kyropoulos growers (a)(b), a Tl doped NaI crystal (c).

\section{Grown crystals and measurements of impurities}

CMO and LMO crystals for the AMoRE were grown successfully. We could grow an LMO crystal with a dimension of $\varnothing 50 \mathrm{~mm} \times \mathrm{H} 70 \mathrm{~mm}$. In order to confirm the effectiveness of the impurity removal in the crystal growth, all the samples were measured using ICP-MS. Table 1 shows impurities in raw powders, purified powder and LMO crystal. Impurities of LMO crystal could be reduced due to a segregation effect during the growth. From this result, even lower impurities are expected in a crystal grown with purified powder.

A NaI:Tl crystal with a dimension of $\varnothing 80 \mathrm{~mm} \times \mathrm{H} 50 \mathrm{~mm}$ could be grown successfully for the COSINE. In order to make a uniform $\mathrm{Tl}$ concentration, the crystal has larger diameter than height as shown in Fig. 2 (c). The amount of $\mathrm{Tl}$ in the NaI: Tl crystal was measured to be 0.136 mol\%. The ICP-MS results show that Sr becomes lower but other impurities of NaI:Tl crystal increased compared to those of the raw powder. It means that there was an external contamination during the crystal growth and we need further studies to trace origins of the impurities and measure contamination factors. 
Table 1: ICP-MS results of powders and LMO and NaI:Tl crystals grown at the CUP.

\begin{tabular}{ccccccc}
\hline Sample & $\begin{array}{c}\mathrm{K} \\
(\mathrm{ppb})\end{array}$ & $\begin{array}{c}\mathrm{Sr} \\
(\mathrm{ppb})\end{array}$ & $\begin{array}{c}\mathrm{Ba} \\
(\mathrm{ppb})\end{array}$ & $\begin{array}{c}\mathrm{Pb} \\
(\mathrm{ppb})\end{array}$ & $\begin{array}{c}\mathrm{Th} \\
(\mathrm{ppb})\end{array}$ & $\begin{array}{c}\mathrm{U} \\
(\mathrm{ppb})\end{array}$ \\
\hline $\mathrm{Li}_{2} \mathrm{CO}_{3}$ & 365.6 & 6.4 & 133.8 & 23.1 & $<0.014$ & 0.414 \\
$\mathrm{MoO}_{3}$ & - & 26.3 & 3113 & 99.9 & 0.15 & 8.0 \\
Purified $\mathrm{MoO}_{3}$ & - & 1,670 & 214.2 & 54.5 & $<0.02$ & 0.85 \\
$\mathrm{LMO}$ crystal (CUP) & - & $<0.05$ & 7.3 & $<0.1$ & $<0.008$ & $<0.008$ \\
$\mathrm{NaI}$ & 248 & 19.0 & 2.9 & 39.9 & $<0.006$ & $<0.006$ \\
$\mathrm{NaI}: T 1$ crystal (CUP) & 566 & 2.8 & 4.1 & 42.7 & $<0.006$ & $<0.006$ \\
\hline
\end{tabular}

\section{Summary}

A clean-environment crystal-growing facility is built at CUP to grow ultra-pure scintillation crystals for searches of WIMP and neutrinoless double beta decay of ${ }^{100} \mathrm{Mo}$. We could successfully grow NaI:Tl crystals using a Kyropoulos method, and $\mathrm{CaMoO}_{4}$ and $\mathrm{Li}_{2} \mathrm{MoO}_{4}$ crystals using a Czochralski method.

The impurity levels of the grown crystals and the raw materials were measured using ICPMS and compared with each other. The $\mathrm{Li}_{2} \mathrm{MoO}_{4}$ crystal shows lowered impurity levels after the growing process and an even better crystal is expected to be grown using purified powders in near future. In case of NaI:Tl crystal, however, we need further studies to understand the contamination during the growing process and how to reduce them effectively.

\section{References}

[1] Adhikari, G., et al., Initial performance of the COSINE-100 experiment, The European Physical Journal C 78.2 (2018): 107.

[2] Alenkov, V. et al., Technical Design Report for the AMoRE Ov $\beta \beta$ Decay Search Experiment, arXiV:1512.05957 (2015).

[3] So, J. H., et al., A study of CaMoO4 crystals for the AMoRE experiment, Nuclear Science Symposium and Medical Imaging Conference (NSS/MIC), 2012 IEEE. IEEE, 2012.

[4] Gileva, Olga, et al., Investigation of the molybdenum oxide purification for the AMoRE experiment, Journal of Radioanalytical and Nuclear Chemistry 314.3 (2017): 1695-1700.

[5] Shin, KeonAh, et al., Reduction of the radioactivity in sodium iodide (NaI) powder by recrystallization method, Journal of Radioanalytical and Nuclear Chemistry 317.3 (2018): 13291332. 